MAGNETOHYDRODYNAMICS Vol. 54 (2018), No. 1-2, pp. 45-48

DOI: $10.22364 / \mathrm{mhd} .54 .1-2.8$

\title{
ROTATING MAGNETIC FIELD INDUCED STRUCTURE FORMATION IN A MAGNETIC FLUID EMULSION
}

\author{
A. Kolesnikova, A. Zakinyan \\ Department of General and Theoretical Physics, North-Caucasus Federal University, \\ 1 Pushkin str., 355009 Stavropol, Russia \\ e-Mail: zakinyan.a.r@mail.ru
}

The microstructure formation in a flat layer of magnetic fluid emulsion in water under the action of an in-plane rotating magnetic field has been studied. Several microstructure types have been found. The revealed microstructures were numerically investigated by Brownian dynamics simulations. The appearance of a macroscopic torque in a magnetic fluid emulsion sample associated with the microstructure dynamics in a rotating magnetic field was observed and studied.

Introduction. Processes of formation and growth of clusters from individual particles or components attract great interest in the context of investigation of complex heterogeneous systems. Many efforts have been undertaken to understand the aggregation dynamics of magnetic microparticles dispersed in a fluid (magnetorheological suspension) and subjected to steady and/or alternating magnetic fields. The studies made [1-3] can be cited as a few examples of research in this direction. The particles under the action of an applied magnetic field undergo an induced complex self-organization process. In the context of the present research, the ordering processes under a rotating uniform magnetic field are of particular interest. In our case, the system under study is a coarse magnetic fluid-in-water (aqueous) emulsion.

The dynamics of microstructure formation in composite materials can manifest themselves in macroscopic mechanical effects. Thus, the rotating clusters, forming in a magnetically active suspension under the action of a rotating magnetic field, can produce a macroscopic torque in a suspension sample. Previously, the effects of internal rotations have been studied with regard to pure magnetic fluids [4-7]. It can be expected that the internal rotation effect will be even more pronounced in a magnetic fluid emulsion than in a pure magnetic fluid.

1. Microstructure of magnetic fluid emulsion. The magnetic fluid emulsion under study was produced by dispersing a kerosene-based magnetic fluid with magnetite nanoparticles in distilled water with an emulsifier by means of ultrasonic disperser. An emulsion with droplet's sizes of few tens of micrometers was produced. The microstructure formation in a flat horizontal layer of such emulsions under the action of an in-plane rotating magnetic field was observed and studied by optical microscopy. A uniform rotating magnetic field was induced by two pairs of perpendicular Helmholtz coils.

Several microstructure types have been found. At comparatively low emulsion concentrations, two types of clusters have been revealed: chain-like aggregates and disc-like clusters (Fig. 1). At low magnetic field frequencies and high magnetic field strengths chain-like structures appear. At higher frequencies and lower magnetic fields droplet discs are formed. The structures evolve in time and their type can change. Accordingly, the initial chains can transform into discs.

The emulsion concentration is also an essential factor for the microstructure formation. It has been found that at comparatively high concentrations the meso- 


\section{A. Kolesnikova, A. Zakinyan}

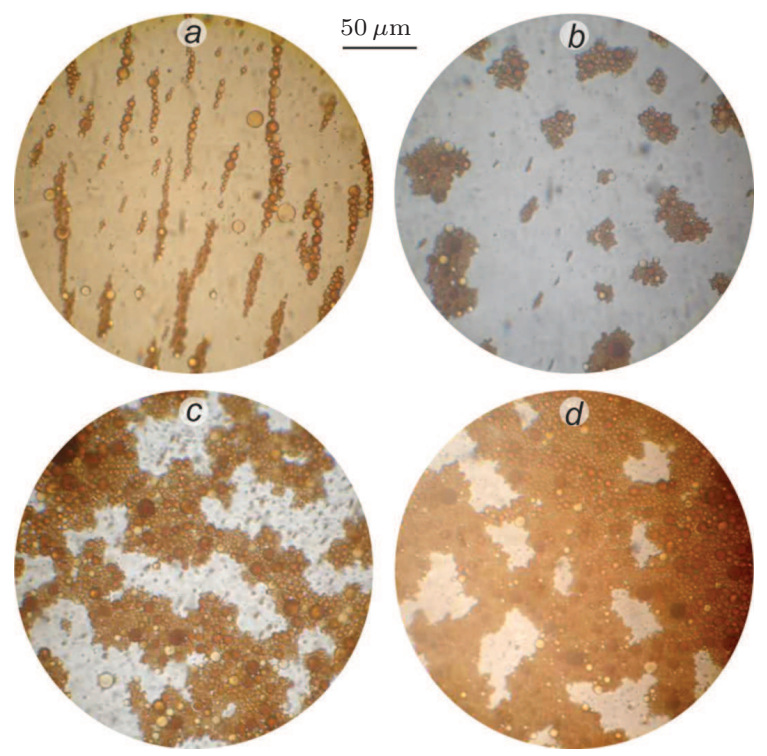

Fig. 1. Microstructures in a magnetic fluid emulsion exposed to a rotating magnetic field. At a low emulsion volume concentration: (a) chain microstructure $(f=1 \mathrm{~Hz}$, $H=9 \mathrm{kA} / \mathrm{m}) ;(b)$ disc-like microstructure $(f=7 \mathrm{~Hz}, H=7.8 \mathrm{kA} / \mathrm{m}) .10$ At a high emulsion volume concentration: $(a)$ branching and $(b)$ occupying structures.
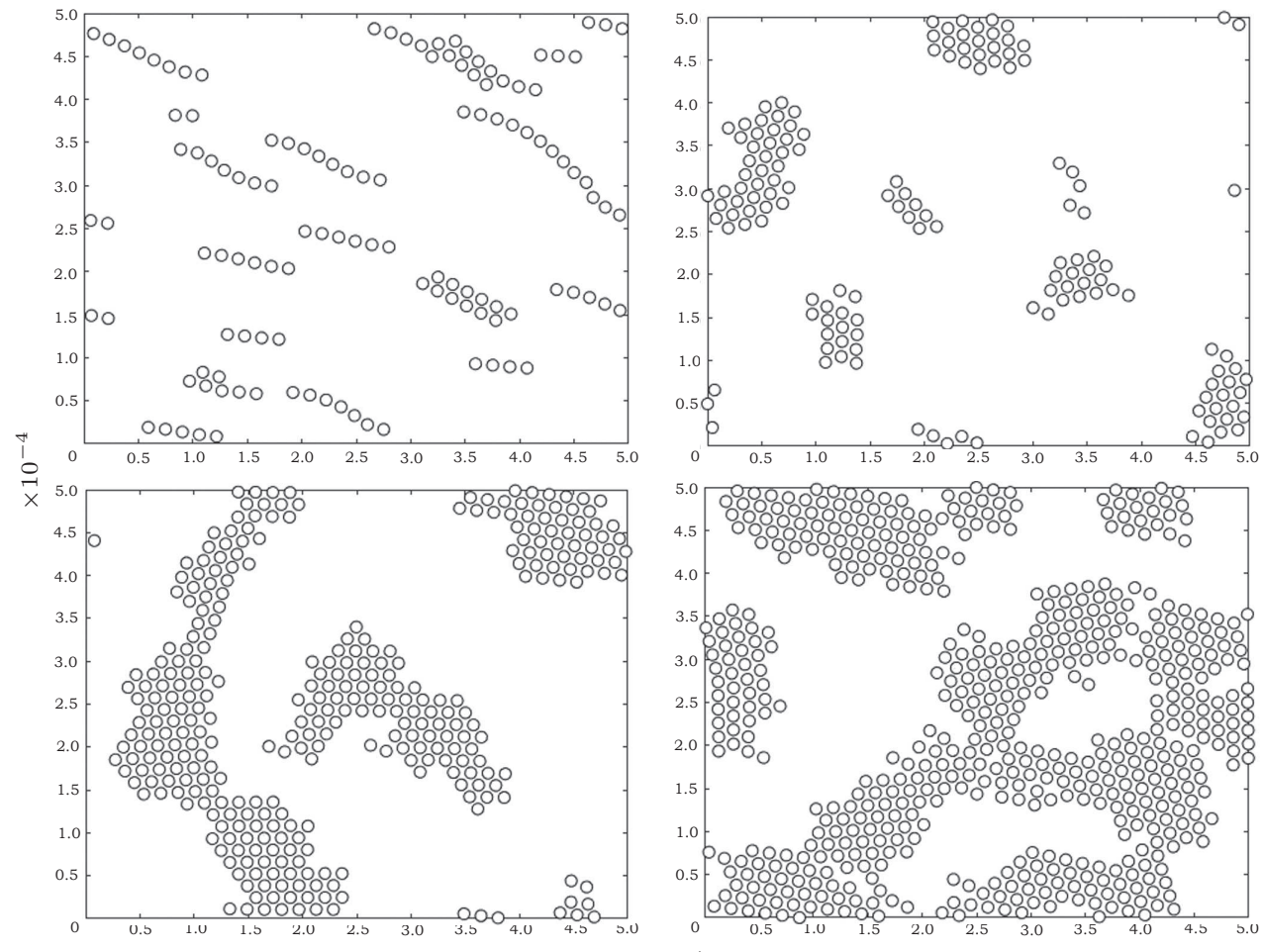

Fig. 2. Results of Brownian dynamic simulations of the microstructure of magnetic fluid emulsions exposed to a rotating magnetic field. Chain-like and disc-like aggregates, branching and occupying structures can be observed. 
scale structures of particle clusters are formed under the action of a rotating magnetic field independently on its frequency and strength. Fig. $1 c$ shows branching structures formed at medium concentrations. At higher concentrations occupying structures occur (Fig. 1d).

The studied systems have been numerically investigated by Brownian dynamics simulations. The structures similar to those observed experimentally were obtained in the calculations with the use of experimental values of the system parameters. The results of the calculations are illustrated in Fig. 2. The observed similarity enables to conclude that the main interactions that characterize the general behavior of the emulsion droplets are the dipolar magnetic interaction, the drag force and the force from the excluded volume of the drops.

2. Macroscopic torque. We observed the appearance of a macroscopic torque in a magnetic fluid emulsion sample in a rotating magnetic field. To measure the torque, the emulsion sample was placed into a hollow plastic sphere which is neutrally buoyanced in the surrounding fluid. Under the action of a uniform rotating magnetic field the sphere rotation was observed. The sphere rotation frequency was measured, from which the toque acting on the sphere can be easily determined. The macroscopic torque generated inside the sphere under a rotating magnetic field due to the ordering processes is balanced by a viscous torque acting, in this case, outside the surrounding fluid.

The macroscopic torque was measured as a function of the magnetic field strength and frequency. The results of the measurements are illustrated in Fig. 3. As seen, the torque grows with increasing magnetic field strength and frequency.

3. Conclusions. Thus, in the present work, the processes of structure formation in a magnetic fluid emulsion have been studied experimentally. Different microstructure types determined by the magnetic field strength, frequency and emulsion concentration were observed and studied. Chain-like, disc-like, branch-

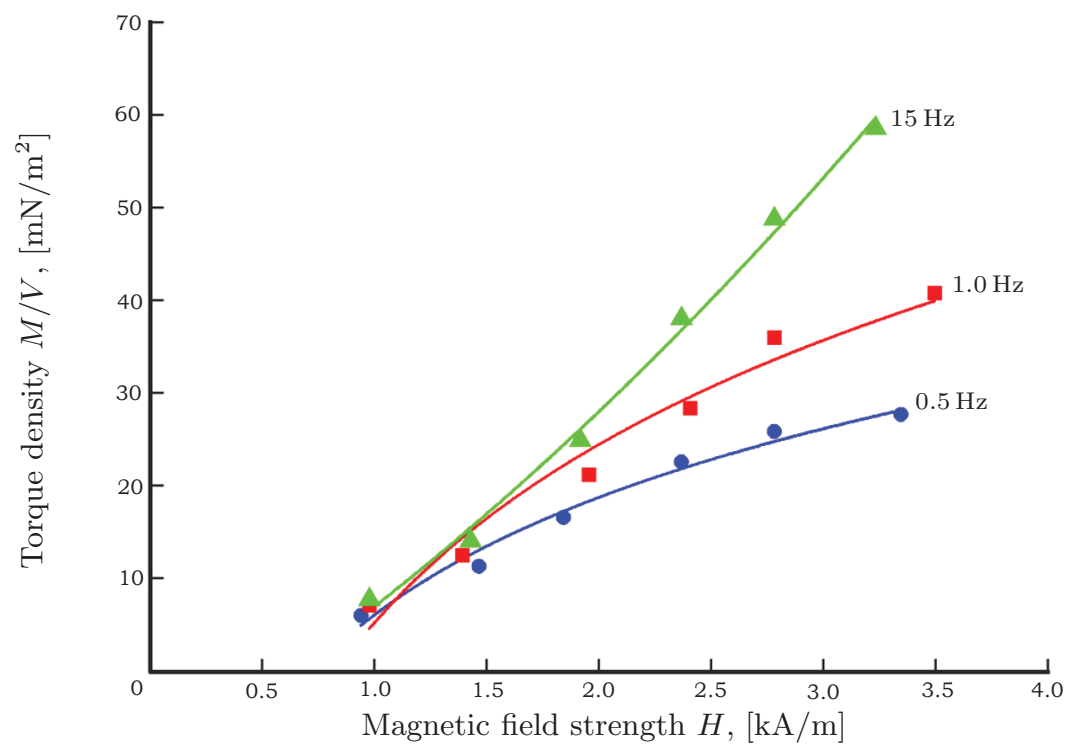

Fig. 3. Macroscopic torque density as a function of the magnetic field strength at different values of the magnetic field frequency. 


\section{A. Kolesnikova, A. Zakinyan}

ing and occupying structures have been analyzed. Similar structures are obtained in the numerical simulations. The appearance of a macroscopic torque in the sample ferrofluid emulsion due to the internal rotations of the formed microstructure elements was observed. It can be concluded that the magnetic fluid emulsions

can be regarded as controllable soft magnetic materials.

4. Acknowledgments. The work was supported by the grant of the President of the Russian Federation No. MK-3169.2017.2 and also by the Ministry of Education and Science of the Russian Federation in the framework of the base part of the governmental ordering for scientific research (project No. 3.5822.2017/8.9).

\section{References}

[1] L.D. Yépez, J.L. Carrillo, F. Donado, J.M. Sausedo-Solorio, P. MiRANDA-Romagnoli. Dynamical pattern formation in a low-concentration magnetorheological fluid under two orthogonal sinusoidal fields. J. Magn. Magn. Mater., vol. 408 (2016), pp. 321-329.

[2] M. Llera, J. Codnia, G.A. Jorge. Aggregation dynamics and magnetic properties of magnetic micrometer-sized particles dispersed in a fluid under the action of rotating magnetic fields. J. Magn. Magn. Mater., vol. 384 (2015), pp. 93-100.

[3] D.V. Gladkikh, Yu.I. Dikansky, A.A. Kolesnikova. Structural transformations in a thin layer of magnetic colloid with magnetized aggregates exposed to an elliptically polarized rotating magnetic field. Magnetohydrodynamics, vol. 52 (2016), no. 3, pp. 319-332.

[4] M.I. Shliomis. Effective viscosity of magnetic suspensions. Sov. Phys. JETP, vol. 34 (1972), pp. 1291-1294.

[5] A.V. Lebedev, A.F. Pshenichnikov. Motion of a magnetic fluid in a rotating magnetic field. Magnetohydrodynamics, vol. 27 (1991), no. 1, pp. 4-8.

[6] A. Chaves, M.Zahn, C. Rinaldi. Spin-up flow of ferrofluids: Asymptotic theory and experimental measurements. Phys. Fluids, vol. 20 (2008), p. 053102 .

[7] I. Torres-Diaz, A. Cortes, Y. Cedeño-Mattei, O. Perales-Perez, C. RinALDI. Flows and torques in Brownian ferrofluids subjected to rotating uniform magnetic fields in a cylindrical and annular geometry. Phys. Fluids, vol. 26 (2014), p. 012004. 